\title{
Analysis of studies of the effectiveness of a biological method of protection for organic crops
}

\author{
Maria Kozłowska ${ }^{1}$, Monika Jaskulska ${ }^{2}$, Agnieszka Lacka ${ }^{1}$, \\ Radosław J. Kozłowski ${ }^{3}$
}

\begin{abstract}
${ }^{1}$ Department of Mathematical and Statistical Methods, Poznan University of Life Sciences, Wojska Polskiego 28, 60-637 Poznań, Poland, e-mail: markoz@up.poznan.pl ${ }^{2}$ Institute of Plant Protection - NRI, W. Węgorka 20, 60-318 Poznań, Poland, ${ }^{3}$ Institute of Agricultural Engineering, Department of Applied IT, Poznan University of Life Sciences, Wojska Polskiego 50, 60-637 Poznań, Poland
\end{abstract}

\begin{abstract}
SUMMARY
As a result of intensive changes in plant production and of environmental changes in agrocenoses, certain agrophages, such as slugs, have in recent years been causing ever increasing amounts of damage to crops of agricultural, horticultural and orchard plants. As a result of the European Parliament and Council Directive adopted on 13 January 2009, the countries of Europe have been required to implement integrated plant protection. One of the principles of this protection is the implementation of strategies which minimize the use of chemical pesticides. Moreover, in accordance with decisions of the European Commission, many active substances have been or are to be withdrawn from the list of available pesticides. An example is metaldehyde, which is to be withdrawn from use in EU countries in the next few years (Commission Decision $\mathrm{C} / 2008 / 7637$ of 5 December 2008). It therefore becomes necessary to seek environmentally safe substances and to develop non-chemical methods for protecting plants against harmful slugs.

An alternative to chemical molluscicides is the parasitic nematode Phasmarhabditis hermaphrodita. Studies have been made of the effectiveness of this biological method in combating Polish populations of the invasive slug species Arion lusitanicus. This was an incomplete multivariate study, i.e. not all variables could be observed for all treatments on all experimental units. Multivariate model of observations has been derived and a method of analysis has been described. The results of these studies provide a basis for the development of environmentally friendly methods of protecting plants against harmful slugs.
\end{abstract}

Key words: incomplete multivariate experiment, derived model of observations, estimation, integrated plant protection 


\section{Introduction}

Agricultural and horticultural plants are often damaged by slugs. One of these is the invasive slug Arion lusitanicus Mabille, 1868 (A. vulgaris Moquin Tandom, 1885) (Anderson, 2005), which originates from the south-western part of Europe. Year by year, in Poland and all over Europe, significant crop damage is being caused by slugs Arion lusitanicus (Kozłowski, 2012; Kozłowski and Kozłowska, 2009; Kozłowski and Kozłowski, 2011). Observations have shown that the losses to crops caused by this slug are large, sometimes as high as $70 \%$. One of the commonest methods of combating slugs is a chemical method involving the use of granulated molluscicides. The active substances of these agents are methiocarb or metaldehyde, which act on slugs gastrically and by contact. Because of its toxicity to some invertebrates and vertebrates, metaldehyde is to be withdrawn from use. An alternative to chemical agents is to use a biological method, involving the use of the parasitic nematode Phasmarhabditis hermaphrodita, of the family Rhabditidae. This is a component of a commercial biological product called Nemaslug (Becker Underwood, UK), which is used to combat various slugs in many European countries (Rae et al., 2008). In the soil the nematodes react with the mucus and excreta of slugs (Rae et al., 2006), and penetrate to the hollow at the end of the slug's mantle (Tan and Grewal, 2001). After penetrating to the interior of the slug the nematodes release the bacterium Moraxella osloensis, which is toxic to the slug. The nematodes feed on bacteria which are present on dead slugs. A new generation of $P$. hermaphrodita then infects other slugs.

The goal of the present research was to obtain information on the effectiveness of the biological product Nemaslug against a Polish population of the slug A. lusitanicus under a variable size of slug population. The fact that this was an incomplete multivariate study, i.e. not all variables could be observed for all treatments on all experimental units, made it necessary to define a model of observations and an estimation of linear parametric functions. 


\section{Materials and methods}

The tests were carried out in laboratory conditions at air temperature $16^{\circ} \mathrm{C}, \mathrm{RH}$ $93 \% \pm 3 \%$ and length of day $12 \mathrm{~h}$. In containers one-third full with soil, six Chinese cabbage plants (Hilton variety) were planted in each container, at the 3-4 leaf stage. The plants in the containers were sprayed once with a solution of $P$. hermaphrodita nematodes in a dose of 30 or 15 nematodes per $\mathrm{cm}^{2}$, and an inclusive control was used in which the plants were sprayed with water (factor A with three levels). Two A. lusitanicus slugs were placed in each container; the slugs had been starved for 48 hours, and had average body mass of $1.002 \mathrm{~g}$. At the next stage of the experiment, in cycles of 5, 10, 15 and 20 days after the application of the nematodes (factor B with four levels), the damaged plants were removed, and six healthy Chinese cabbage seedlings were placed in each container, as well as one A. lusitanicus slug. Three replications were applied. The percentage of plant damage was observed according to a 5-point scale $(0,25,50,75$ and $100 \%$ damaged plant surface area), and records were made of healthy, sick and dead slugs. Observations of the percentage of plant damage were carried out on the newly introduced healthy seedlings every 10 days, from the first day after the application of nematodes until the 61st day.

The scheme of cyclical changes is presented in Figure 1. After the first day, following application of the Nemaslug biological product, observations were made of damage done to the Chinese cabbage seedlings by the two slugs grazing in each container, for all $a b=12$ experimental combinations. After 10 and 50 days the healthy seedlings were placed in containers with combinations of levels $\mathrm{B}_{1}$ and $\mathrm{B}_{2}$ (cycle of changes after 5 and 10 days). Thus on the 11th and 51st day after application of Nemaslug it was not possible to make observations for the other combinations. After 20 and 40 days the seedlings were replaced in containers with combinations of the levels $\mathrm{B}_{1}, \mathrm{~B}_{2}$ and $\mathrm{B}_{4}$ (cycle of changes after 5, 10 and 20 days). After 30 days healthy seedlings were placed in containers with combinations of levels $\mathrm{B}_{1}, \mathrm{~B}_{2}$ and $\mathrm{B}_{3}$ (cycle of changes after 5, 10 and 15 days). Finally after 60 days the seedlings were changed for all combinations 
(5, 10, 15 and 20 days). The scheme of cyclical changes implies the incompleteness of the experimental design, determining the impossibility of observing certain variables from the set of $t=7$ variables for certain treatments among the twelve experimental combinations.

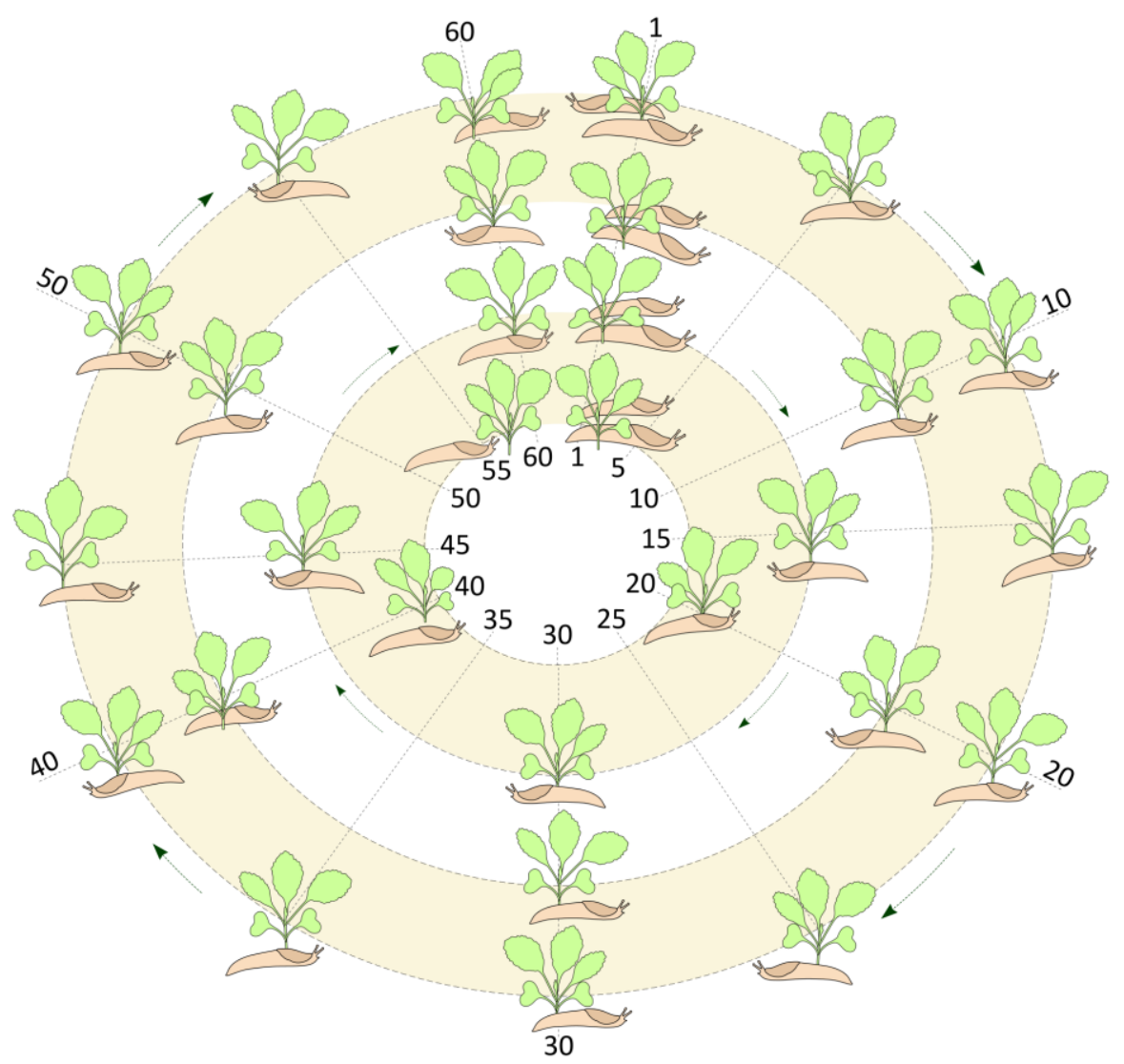

Figure 1. The scheme of cyclical changes; picture of plant and slug denote placement of healthy seedlings and slug in experimental unit, respectively

Let us write down the model of observations. For each cycle of changes (level $\mathrm{B}_{\mathrm{i}}, i=1,2, \ldots, b$, here $b=4$ ) the model of observations is of the form

$$
\mathbf{Y}^{(i)}=\mathbf{1}_{a r}\left(\boldsymbol{\mu}^{(i)}\right)^{\prime}+\left(\mathbf{I}_{a} \otimes \mathbf{1}_{r}\right) \boldsymbol{\gamma}^{(i)}+\boldsymbol{\varepsilon}^{(i)},
$$


where $\mathbf{Y}^{(i)}$ is an $\operatorname{ar} \times t_{i}$ matrix of observations for combinations of the $i$ th level of factor B and all $a$ levels of factor A (here $a=3$ ), $\boldsymbol{\mu}^{(i)}$ jest is a $t_{i}$-dimensional vector of common values, $\gamma^{(i)}$ is an $a \times t_{i}$ matrix of object effects (here the combinations $\mathrm{A}_{1} \mathrm{~B}_{\mathrm{i}}, \mathrm{A}_{2} \mathrm{~B}_{\mathrm{i}}, \mathrm{A}_{3} \mathrm{~B}_{\mathrm{i}}$ ), and $\boldsymbol{\varepsilon}^{(i)}$ is an appropriate matrix of random errors, where the expected value of $\boldsymbol{\varepsilon}^{(i)}$ is the zero matrix, and the dispersion matrix for $\operatorname{cs} \boldsymbol{\varepsilon}^{(i)}$ (column string of $\boldsymbol{\varepsilon}^{(i)}$ ) is the Kronecker product of an unknown $t_{i} \times t_{i}$ dispersion matrix $\Sigma^{(i)}$ for each row of the matrix $\boldsymbol{\varepsilon}^{(i)}$ and the identity matrix of degree $a r$. Because the matrix of object parameters can be written in the form $\boldsymbol{\gamma}^{(i)}=\boldsymbol{\alpha}^{(i)}+\mathbf{1}_{a}\left(\boldsymbol{\beta}^{(i)}\right)^{\prime}+(\boldsymbol{\alpha} \boldsymbol{\beta})^{(i)}$, where $\boldsymbol{\alpha}^{(i)}$ is an $a \times t_{i}$ matrix of main effects of factor $\mathrm{A}, \boldsymbol{\beta}^{(i)}$ is a $t_{i}$-dimensional vector of main effects of level $\mathrm{B}_{\mathrm{i}}$ of factor $\mathrm{B}$, and $(\boldsymbol{\alpha} \boldsymbol{\beta})^{(i)}$ is an $a \times t_{i}$ matrix of interaction effects of the $i$ th level of factor $\mathrm{B}$ with levels of factor $\mathrm{A}$. We therefore have a model of observations in the form

$$
\mathbf{Y}^{(i)}=\mathbf{1}_{a r}\left(\boldsymbol{\mu}^{(i)}\right)^{\prime}+\left(\mathbf{I}_{a} \otimes \mathbf{1}_{r}\right)\left(\boldsymbol{\alpha}^{(i)}+\mathbf{1}_{a}\left(\boldsymbol{\beta}^{(i)}\right)^{\prime}+(\boldsymbol{\alpha} \boldsymbol{\beta})^{(i)}\right)+\boldsymbol{\varepsilon}^{(i)} .
$$

which can also be written as follows:

$$
\mathbf{Y}^{(i)}=\mathbf{1}_{a r}\left(\boldsymbol{\mu}^{(i)}\right)^{\prime}+\left(\mathbf{I}_{a} \otimes \mathbf{1}_{r}\right) \boldsymbol{\alpha}^{(i)}+\mathbf{1}_{a r}\left(\boldsymbol{\beta}^{(i)}\right)^{\prime}+\left(\mathbf{I}_{a} \otimes \mathbf{1}_{r}\right)(\boldsymbol{\alpha} \boldsymbol{\beta})^{(i)}+\boldsymbol{\varepsilon}^{(i)} .
$$

We now introduce the $t \times t_{i}$ matrix $\mathbf{M}_{\mathrm{i}}$, whose $\left(l, l_{i}\right)$-th element is 1 when the $l$ th variable is observed in the $i$ th group at number $l_{i},\left(l=1,2, \ldots, t ; l_{i}=1,2, \ldots, t_{i}\right)$, and zero otherwise. This matrix is of full column rank; for every $i, \mathrm{r}\left(\mathbf{M}_{\mathrm{i}}\right)=t_{i}$. We can also write $\left(\boldsymbol{\mu}^{(i)}\right)^{\prime}=\boldsymbol{\mu} \mathbf{M}_{i}$ and $\boldsymbol{\alpha}^{(i)}=\boldsymbol{\alpha} \mathbf{M}_{i}$, where $\boldsymbol{\mu}$ and $\boldsymbol{\alpha}$ are defined for the whole experimental design, $\boldsymbol{\mu}$ being a $t$-dimensional vector of common values, while $\boldsymbol{\alpha}$ is an $a \times t$ matrix of main effects of factor A. The matrix $\mathbf{M}_{\mathrm{i}}$ indicates those elements of the vector of common values $\boldsymbol{\mu}$ and the matrix $\boldsymbol{\alpha}$ of main effects of factor A, respectively, which correspond to the variables observed in the $i$ th subset of experimental units. Moreover the dispersion matrix for $\operatorname{cs} \boldsymbol{\varepsilon}^{(i)}$ can be written in the form $\boldsymbol{\Sigma}^{(i)} \otimes \mathbf{I}_{a r}=\mathbf{M}_{i} \boldsymbol{\Sigma} \mathbf{M}_{i} \otimes \mathbf{I}_{a r}$, where $\boldsymbol{\Sigma}$ is a $t \mathbf{x} t$ unknown non-singular dispersion matrix defined for the overall design.

The matrix of all observations for the entire experimental design is of the form 


$$
\begin{aligned}
& {\left[\begin{array}{c}
\mathbf{Y}^{(1)} \mathbf{M}_{1}^{\prime} \\
\mathbf{Y}^{(2)} \mathbf{M}_{2}^{\prime} \\
\vdots \\
\mathbf{Y}^{(b)} \mathbf{M}_{b}^{\prime}
\end{array}\right]=\left[\begin{array}{c}
\mathbf{1}\left(\boldsymbol{\mu}^{(1)}\right)^{\prime} \mathbf{M}_{1}^{\prime} \\
\mathbf{1}\left(\boldsymbol{\mu}^{(2)}\right)^{\prime} \mathbf{M}_{2}^{\prime} \\
\vdots \\
\mathbf{1}\left(\boldsymbol{\mu}^{(b)}\right)^{\prime} \mathbf{M}_{b}^{\prime}
\end{array}\right]+\left[\begin{array}{c}
\left(\mathbf{I}_{a} \otimes \mathbf{1}_{r}\right)\left(\boldsymbol{\alpha}^{(1)}\right)^{\prime} \mathbf{M}_{1}^{\prime} \\
\left(\mathbf{I}_{a} \otimes \mathbf{1}_{r}\right)\left(\boldsymbol{\alpha}^{(2)}\right)^{\prime} \mathbf{M}_{2}^{\prime} \\
\vdots \\
\left(\mathbf{I}_{a} \otimes \mathbf{1}_{r}\right)\left(\boldsymbol{\alpha}^{(b)}\right)^{\prime} \mathbf{M}_{b}^{\prime}
\end{array}\right]+\left[\begin{array}{cccc}
\mathbf{1}_{a r} & \mathbf{0} & \cdots & \mathbf{0} \\
\mathbf{0} & \mathbf{1}_{a r} & \cdots & \mathbf{0} \\
\vdots & \vdots & \ddots & \vdots \\
\mathbf{0} & \mathbf{0} & \cdots & \mathbf{1}_{a r}
\end{array}\right]} \\
& \cdot\left[\begin{array}{c}
\left(\boldsymbol{\beta}^{(1)}\right)^{\prime} \mathbf{M}_{1}^{\prime} \\
\left(\boldsymbol{\beta}^{(2)}\right)^{\prime} \mathbf{M}_{2}^{\prime} \\
\vdots \\
\left(\boldsymbol{\beta}^{(b)}\right)^{\prime} \mathbf{M}_{b}^{\prime}
\end{array}\right]+\left[\begin{array}{cccc}
\mathbf{I}_{a} \otimes \mathbf{1}_{r} & \mathbf{0} & \cdots & \mathbf{0} \\
\mathbf{0} & \mathbf{I}_{a} \otimes \mathbf{1}_{r} & \cdots & \mathbf{0} \\
\vdots & \vdots & \ddots & \vdots \\
\mathbf{0} & \mathbf{0} & \cdots & \mathbf{I}_{a} \otimes \mathbf{1}_{r}
\end{array}\right]\left[\begin{array}{c}
(\boldsymbol{\alpha} \boldsymbol{\beta})^{(1)} \mathbf{M}_{1}^{\prime} \\
(\boldsymbol{\alpha} \boldsymbol{\beta})^{(2)} \mathbf{M}_{2}^{\prime} \\
\vdots \\
(\boldsymbol{\alpha} \boldsymbol{\beta})^{(b)} \mathbf{M}_{b}^{\prime}
\end{array}\right]+\left[\begin{array}{c}
\boldsymbol{\varepsilon}^{(1)} \mathbf{M}_{1}^{\prime} \\
\boldsymbol{\varepsilon}^{(2)} \mathbf{M}_{2}^{\prime} \\
\vdots \\
\boldsymbol{\varepsilon}^{(b)} \mathbf{M}_{b}^{\prime}
\end{array}\right]
\end{aligned}
$$

while the dispersion matrix for uncorrelated random errors $\boldsymbol{\varepsilon}^{(i)} \mathbf{M}_{i}^{\prime}$ is of the form $\mathbf{M}_{i} \boldsymbol{\Sigma}^{(i)} \mathbf{M}_{i}^{\prime} \otimes \mathbf{I}_{a r}=\mathbf{M}_{i} \mathbf{M}_{i}^{\prime} \boldsymbol{\Sigma} \mathbf{M}_{i} \mathbf{M}_{i}^{\prime} \otimes \mathbf{I}_{a r}$.

In the considered experiment $b=4$ and the matrices $\mathbf{M}_{\mathrm{i}}$ have the form $\mathbf{M}_{1}=\mathbf{M}_{2}=\mathbf{I}_{7}$,

$$
\mathbf{M}_{3}^{\prime}=\left[\begin{array}{lllllll}
1 & 0 & 0 & 0 & 0 & 0 & 0 \\
0 & 0 & 0 & 1 & 0 & 0 & 0 \\
0 & 0 & 0 & 0 & 0 & 0 & 1
\end{array}\right] \text { and } \mathbf{M}_{4}^{\prime}=\left[\begin{array}{lllllll}
1 & 0 & 0 & 0 & 0 & 0 & 0 \\
0 & 0 & 1 & 0 & 0 & 0 & 0 \\
0 & 0 & 0 & 0 & 1 & 0 & 0 \\
0 & 0 & 0 & 0 & 0 & 0 & 1
\end{array}\right] .
$$

The matrix of treatment effects is of the form $\boldsymbol{\gamma}=\left[\left(\boldsymbol{\gamma}^{(1)} \mathbf{M}_{1}^{\prime}\right)^{\prime}\left(\boldsymbol{\gamma}^{(2)} \mathbf{M}_{2}^{\prime}\right)^{\prime}\left(\boldsymbol{\gamma}^{(3)} \mathbf{M}_{3}^{\prime}\right)^{\prime}\left(\boldsymbol{\gamma}^{(4)} \mathbf{M}_{4}^{\prime}\right)^{\prime}\right]^{\prime}$. The estimation of treatment parameter functions as presented in Appendix can be applied here, although the incompleteness of the design means that for a given vector $\mathbf{d}$ not all functions $\mathbf{c}^{\prime} \boldsymbol{\gamma d}$ can be estimated. Let $\mathbf{c}^{\prime} \mathbf{1}=0$; then the function $\mathbf{c}^{\prime} \boldsymbol{\gamma d}$ is called a contrast. We define the following sets of vectors:

$$
\begin{aligned}
& A=\left\{\mathbf{c}^{\prime}: \mathbf{c}^{\prime} \mathbf{1}=0\right\}, B=\left\{\mathbf{c}^{\prime}: \mathbf{c}^{\prime}=\left[\mathbf{c}_{[1]}^{\prime}, \mathbf{c}_{[2]}^{\prime}, \mathbf{0}^{\prime}\right], \underset{\mathbf{c}_{[\bullet]} \in M_{3}}{\forall} \mathbf{c}^{\prime} \mathbf{1}=0\right\}, \\
& C=\left\{\mathbf{c}^{\prime}: \mathbf{c}^{\prime}=\left[\mathbf{c}_{[3]}^{\prime}, \mathbf{0}^{\prime}, \mathbf{c}_{[4]}^{\prime}\right], \underset{\mathbf{c}_{[\bullet]} \in M_{3}}{\forall} \mathbf{c}^{\prime} \mathbf{1}=0\right\}, \\
& D=\left\{\mathbf{c}^{\prime}: \mathbf{c}^{\prime}=\left[\mathbf{c}_{[5]}^{\prime}, \mathbf{c}_{[6]}^{\prime}, \mathbf{c}_{[7]}^{\prime}, \mathbf{0}^{\prime}\right], \underset{\mathfrak{c}_{[\bullet]} \in M_{3}}{\forall} \mathbf{c}^{\prime} \mathbf{1}=0\right\},
\end{aligned}
$$


where $M_{s}$ denotes a set of $s$-dimensional real vectors. We note that $B=C \cap D \subset A, B \subset C, B \subset D, B, C, D \subset A$. For a selected vector $\mathbf{d}$ there can be estimated all contrasts with a vector of coordinates from one of the above sets.

For example

for the vectors $[1,0,0,0,0,0,0]^{\prime}$ and $[0,0,0,0,0,0,1]^{\prime}-$ from set $A$,

for the vectors $[0,1,0,0,0,0,0]^{\prime}$ and $[0,0,0,0,0,1,0]^{\prime}-$ from set $B$,

for the vectors $[0,0,1,0,0,0,0]^{\prime}$ and $[0,0,0,0,1,0,0]^{\prime}-$ from set $C$,

for the vector $[0,0,0,1,0,0,0]^{\prime}-$ from set $D$,

from which it follows that, with respect to each variable and all variables simultaneously, it is possible to compare the effectiveness of doses of nematode solution with a control under a variable size of slug population conditioned by the five-day and ten-day cycle of changes (contrasts defined by vectors from set $B$ ). According to theory presented in Appendix approximate $\mathrm{F}$ test can be applied now for the testing particular hypotheses.

\section{Results and discussion}

For over a decade various data have been published in the literature concerning the biological use of $P$. hermaphrodita to combat various slugs in the Gastropoda: Pulmonata group. In 1994 that nematode was made commercially available for the first time as a biological agent for combating slugs (Glen et al., 1996). The nematodes infect the slugs fairly rapidly, leading to reduction in grazing on plants, and to the death of slugs. According to Speiser et al. (2001), A. lusitanicus and D. reticulatum are the most harmful species in Switzerland. Those authors evaluated their sensitivity to infection by $P$. hermaphrodita in laboratory conditions. They observed that $D$. reticulatum was strongly infected by the nematodes, irrespective of the slugs' body mass, while in A. lusitanicus only slugs at a young stage of development were infected.

In the present study, basic contrasts were estimated for selected variables, e.g. contrasts between the main effects of a factor whose levels were doses of a solution of $P$. hermaphrodita nematodes (for $\mathbf{c}_{1}^{\prime}=\mathbf{1}_{4}{ }^{\prime} \otimes[1,1,-2] \in A$ and 
$\mathbf{d}_{1}=\left[\mathbf{0}_{\mathbf{6}}{ }^{\prime}, 1\right]^{\prime} ; \quad$ for $\quad \mathbf{c}_{2}^{\prime}=\left[\mathbf{1}_{2}^{\prime} \otimes[1,1,-2] \quad \mathbf{0}_{\mathbf{6}}{ }^{\prime}\right] \in B \quad$ for all variables; for $\mathbf{c}_{3}^{\prime}=\mathbf{1}_{4}{ }^{\prime} \otimes[1,-1,0] \in A \quad$ and $\quad \mathbf{d}_{1}=\left[\mathbf{0}_{\mathbf{6}^{\prime}}, 1\right]^{\prime} ; \quad$ for $\quad \mathbf{c}_{4}^{\prime}=\left[\mathbf{1}_{2}{ }^{\prime} \otimes[1,-1,0] \mathbf{0}_{\mathbf{6}}{ }^{\prime}\right] \in B$ for all variables). Differences were found between the mean of damage done to plants which had been sprayed with the nematode solution and to the control plants, which confirmed the effectiveness of the analysed solution at doses of both 30 and 15 nematodes per $\mathrm{cm}^{2}$ (Tab. 1). The nematodes infected the slugs, and consequently caused a reduction in their grazing on plants.

Table 1. Estimate of contrasts of the twelve experimental combinations for observed variables

\begin{tabular}{|c|c|c|c|c|c|c|c|}
\hline \multirow[t]{2}{*}{ Contrast } & \multicolumn{7}{|c|}{ Variable } \\
\hline & 1 & 2 & 3 & 4 & 5 & 6 & 7 \\
\hline $\mathbf{c}_{1}^{\prime}=\mathbf{1}_{4}^{\prime} \otimes[1,1,-2] \in A$ & & & & & & & $-106,9$ \\
\hline $\mathbf{c}_{2}^{\prime}=\left[\mathbf{1}_{2}^{\prime} \otimes[1,1,-2] \mathbf{0}_{\mathbf{6}^{\prime}}{ }^{\prime}\right] \in B$ & $-1,4$ & $-26,4$ & $-45,8$ & 12,5 & $-32,0$ & 33,3 & $-77,8$ \\
\hline $\mathbf{c}_{3}^{\prime}=\mathbf{1}_{4}^{\prime} \otimes[1,-1,0] \in A$ & & & & & & & $-45,8$ \\
\hline $\mathbf{c}_{4}^{\prime}=\left[\mathbf{1}_{2}^{\prime} \otimes[1,-1,0] \mathbf{0}_{6}{ }^{\prime}\right] \in B$ & $-9,7$ & $-15,3$ & $-15,3$ & $-15,3$ & $-23,6$ & $-25,0$ & $-38,9$ \\
\hline
\end{tabular}

Experiments on Chinese cabbage have also been carried out by researchers at Aberdeen University in Scotland. Field studies on several species of slugs were carried out on experimental plots using different methods of application of $P$. hermaphrodita. It was found that on the 14th day of the experiment, nematodes applied three times in a quantity of one-sixth of the recommended dose of $P$. hermaphrodita ( 5 nematodes per $\mathrm{cm}^{2}$ ), and nematodes applied around the base of plants (3000 nematodes per plant) protected the plants from slug damage. On the 28th and 35th day all nematode treatments brought a reduction in the damage caused by slugs relative to the control plants, although after weeks of duration of the experiment that difference became much smaller (Rae et al., 2009).

In the present study, comparing the effect of $P$. hermaphrodita on the amount of slug damage to plants after successive cyclical replacements of plants and increase in the number of slugs, the growth in damage was found to be 
differentiated. The quantity of damage to Chinese cabbage on the control units was greater than on the units treated with a solution of $P$. hermaphrodita in both doses. The differences between the quantity of damage done to the control plants and the average damage to plants on units treated following successive cyclical changes was $19 \%$ on average.

Grubisic et al. (2003) describe an experiment on Chinese cabbage seedlings grown under cover, performed in Croatia. They state that after 10-30 days from the application of nematodes and the methiocarb the plants were significantly less damaged by slugs. The respective levels of damage for P. hermaphrodita, methiocarb and the control were $2 \%, 15 \%$ and $40 \%$.

In the present study, after 60 days from the application of nematodes on all treated units, on the newly introduced healthy seedlings there were observed to be "limited" effects of slug grazing with respect to control, as is evidenced by the estimated contrast values $\mathbf{c}_{1}^{\prime} \boldsymbol{\gamma} \mathbf{d}_{1}=-106,9$ (Tab. 1). The durability of the action of the solution of $P$. hermaphrodita nematodes, applied 61 days earlier, was confirmed.

In the case of many reported experiments with the use of infectious nematodes the results are not definitive. In some cases the applied nematodes did not affect the slug death rate, although it affected their grazing (Glen et al., 2000). It is suspected that slugs of the family Arionidae are relatively difficult to infect (Glen et al., 1996).

In the case of use of the biological product Nemaslug, a significant fact is that nematodes may survive approximately six weeks in soil. Vernavá et al. (2004) found that they were still present in the soil after 99 days. For this reason it is not necessary to apply the product multiple times; this brings about significant reduction in the costs of protecting plants against slugs.

In our study the effect of the action of the nematodes was visible up to the last day of the experiment. Under a variable size of population of slugs in the containers, for doses of both 30 and 15 nematodes per $\mathrm{cm}^{2}$, there was observed to be a reduction in the damage done by slugs to successive cyclically introduced healthy Chinese cabbage seedlings. 


\section{Appendix}

For the description of multivariate experiments, multidimensional linear models are used with the form $\mathbf{Y}=\mathbf{X} \Gamma+\mathbf{E}$ (Morrison, 2005), where $\mathbf{Y}$ is an $n \mathbf{x} t$ matrix of observations of $t$ variables on $n$ experimental units, $\mathbf{X}$ is a known $n \times p$ design matrix, $\Gamma$ is a $p \times t$ matrix of unknown parameters, and $\mathrm{E}$ is an $n \times t$ matrix of random errors. It is assumed that the matrix of expected values of matrix $\mathbf{E}$ is the zero matrix, and the dispersion matrix for $\operatorname{csE}$ is the Kronecker product of an unknown $t \mathrm{x} t$ dispersion matrix $\Sigma$ for each row of $\mathrm{E}$ and the identity matrix of degree $n$. The estimability of the linear parametrical functions $\mathbf{b}^{\prime} \boldsymbol{\Gamma} \mathbf{d}$, where $\mathbf{b}$ and $\mathbf{d}$ are arbitrary non-zero vectors, is defined analogously as in the onedimensional linear model. We therefore have the expected value $E\left(\mathbf{a}^{\prime} \mathbf{Y d}\right)=\mathbf{b}^{\prime} \boldsymbol{\Gamma} \mathbf{d}$ and the variance $\operatorname{Var}\left(\mathbf{a}^{\prime} \mathbf{Y d}\right)=\mathbf{a} \mathbf{a}^{\prime} \cdot \mathbf{d}^{\prime} \boldsymbol{\Sigma} \mathbf{d}$, where $\mathbf{a}^{\prime} \mathbf{X}=\mathbf{b}^{\prime}$. From the Gauss-Markov theory of the least squares method, it is known that this variance reaches a minimum only where the estimator of the linear parametric function has the form $\mathbf{b}^{\prime} \boldsymbol{\Gamma} \mathbf{d}=\mathbf{b}^{\prime} \boldsymbol{\Gamma}^{0} \mathbf{d}$, where $\boldsymbol{\Gamma}^{0} \mathbf{d}$ is a solution of the system of normal equations $\mathbf{X}^{\prime} \mathbf{X} \Gamma^{0} \mathbf{d}=\mathbf{X}^{\prime} \mathbf{Y d}$, from which, due to the arbitrary choice of $\mathbf{d}$, it follows that $\Gamma^{0}=\left(\mathbf{X}^{\prime} \mathbf{X}\right)^{-} \mathbf{X}^{\prime} \mathbf{Y}$. The estimator $\mathbf{b}^{\prime} \boldsymbol{\Gamma} \mathbf{d}=\mathbf{b}^{\prime} \boldsymbol{\Gamma}^{0} \mathbf{d}$ is BLUE. Let us note also that, using a vector $\mathbf{d}=\left[d_{1}, d_{1}, \ldots, d_{t}\right]^{\prime}$ where $d_{\mathrm{i}}=1$ and $d_{\mathrm{j}}=0$ for $j \neq i$, it follows from the identity $\mathbf{b}^{\prime} \boldsymbol{\Gamma} \mathbf{d}=\mathbf{b}^{\prime} \boldsymbol{\Gamma}^{0} \mathbf{d}$ and the above considerations that $E\left(\mathbf{b}^{\prime} \boldsymbol{\Gamma}^{0}\right)=\mathbf{b}^{\prime}\left(\mathbf{X}^{\prime} \mathbf{X}\right)^{-} \mathbf{X} \mathbf{X} \boldsymbol{\Gamma}$. Therefore the function $\mathbf{b}^{\prime} \mathbf{\Gamma} \mathbf{d}$ is estimable in the above model if for an arbitrary g-inverse $\left(\mathbf{X}^{\prime} \mathbf{X}\right)^{-}$of the matrix $\mathbf{X}^{\prime} \mathbf{X}$ the condition $\mathbf{b}^{\prime}\left(\mathbf{X}^{\prime} \mathbf{X}\right)^{-} \mathbf{X}^{\prime} \mathbf{X}=\mathbf{b}^{\prime}$ is satisfied.

For multivariate analysis it is often of interest to consider linear parametric functions based only on certain rows of the matrix $\boldsymbol{\Gamma}$. It is then convenient to represent the considered model in the form

$$
\mathbf{Y}=\left[\begin{array}{ll}
\mathbf{X}_{0} & \mathbf{X}_{1}
\end{array}\right] \cdot\left[\begin{array}{l}
\boldsymbol{\eta} \\
\boldsymbol{\gamma}
\end{array}\right]+\mathbf{E},
$$


where $\mathbf{X}_{0}$ and $\mathbf{X}_{1}$ and $\boldsymbol{\eta}$ and $\boldsymbol{\gamma}$ are respectively $n \times p_{1}$ and $n \times p_{2}$ submatrices of the design and $p_{1} \times t$ and $p_{2} \mathrm{x} t$ matrices of parameters for $t$ observed variables. The system of normal equations then takes the form

$$
\left[\begin{array}{ll}
\mathbf{X}_{0}^{\prime} \mathbf{X}_{0} & \mathbf{X}_{0}^{\prime} \mathbf{X}_{1} \\
\mathbf{X}_{1}^{\prime} \mathbf{X}_{0} & \mathbf{X}_{1}^{\prime} \mathbf{X}_{1}
\end{array}\right]\left[\begin{array}{l}
\boldsymbol{\eta} \\
\boldsymbol{\gamma}
\end{array}\right]=\left[\begin{array}{l}
\mathbf{X}_{0}^{\prime} \\
\mathbf{X}_{1}^{\prime}
\end{array}\right] \mathbf{Y} .
$$

If we are interested in linear functions of the parameters contained in the submatrix $\gamma$, the above system of equations is reduced by multiplying through by the matrix $\left[\begin{array}{ll}\mathbf{B} & \mathbf{I}\end{array}\right]$, selecting the $p_{2} \quad \mathbf{x} \quad p_{1}$ matrix $\mathbf{B}$ so that $\left[\mathbf{B X}_{0}^{\prime}+\mathbf{X}_{1}^{\prime}\right] \mathbf{X}_{0}=\mathbf{0}$. A solution of the system of equations reduced in this way is $\boldsymbol{\gamma}^{0}=\left[\left(\mathbf{B} \mathbf{X}_{0}^{\prime}+\mathbf{X}_{1}^{\prime}\right) \mathbf{X}_{1}\right]^{-}\left(\mathbf{B} \mathbf{X}_{0}^{\prime}+\mathbf{X}_{1}^{\prime}\right) \mathbf{Y}$. Thus for $\mathbf{b}^{\prime}=\left[\begin{array}{ll}\mathbf{0}^{\prime} & \mathbf{c}^{\prime}\end{array}\right]$ the estimator of the functions $\mathbf{c}^{\prime} \boldsymbol{\gamma}^{0} \mathbf{d}$ of interest is BLUE.

Let us consider the testing of the general hypothesis $H_{0}: \mathbf{C}_{H} \gamma \mathbf{D}_{H}=\mathbf{0}$, where $\mathbf{C}_{\mathrm{H}}$ is a known matrix of full rank, and $\mathbf{D}_{\mathrm{H}}$ is also known. Because the functions $\mathbf{C}_{H} \boldsymbol{\gamma} \mathbf{D}_{H}$ are estimable, the hypothesis is testable. We decompose the general hypothesis into particular hypotheses $H_{0 k l}: \mathbf{c}_{H k}^{\prime} \gamma \mathbf{d}_{H l}=0$, where $\mathbf{c}_{H k}^{\prime}$ is the $k$ th row of matrix $\mathbf{C}_{\mathrm{H}}$, and $\mathbf{d}_{H l}$ is the $l$ th column of matrix $\mathbf{D}_{\mathrm{H}}$. For the verification of these hypotheses an approximate $\mathrm{F}$ test is proposed (Scheffe, 1959). The rejection of any one of the particular null hypotheses usually entails rejection of the general hypothesis, while non-rejection of all of the particular hypotheses is equivalent to the non-rejection of the general hypothesis.

\section{Acknowledgements}

The project is supported by National Center for Science contract no. 7350/B/P01/2011/40.

\section{REFERENCES}

Anderson R., (2005): An annotated list of the non-marine Mollusca of Britain and Ireland. J. Conch. 38(6): 607-637.

Glen D.M., Wilson M.J., Hughes L., Cargeeg P., Hajjar A. (1996): Exploring and exploiting the potential of the rhabditid nematode Phasmarhabditis hermaphrodita 
as a biocontrol agent for slugs. In: Henderson, I.F. (ed.) Slug and Snail Pests in Agriculture. Monograph No. 66, British Crop Protection Council, Thornton Heath, UK: 271-280

Glen D.M., Wiltshire C.W., Hughes L., Ester A., van Rozen K., Castillejo J., Iglesias J., Speiser B., Coupland J., Gwynn R. (2000): The use of slug-parasitic nematodes and other techniques for control of slug and snail damage in horticultural crops. Brighton Crop Protection Conference (BCPC) Pests and Diseases: 345-350.

Grubisic D., Ostrec L.J., Dusak I. (2003): Biological control of slugs in vegetable crops in Croatia. In: Dussart, G.B.J. (ed.) Slugs \& Snails: Agricultural, Veterinary \& Environmental Perspectives. Monograph No. 80, British Crop Protection Council, Thornton Heath, UK: 115-120.

Kozłowski J. (2012): The significance of alien and invasive slug species for plant communities in agrocenoses. J. Plant Prot. Res. 52(1): 67-76.

Kozłowski J., Kozłowska M. (2009): Palatability and consumption of 95 species of herbaceous plants and oilseed rape for Arion lusitanicus Mabille, 1868. J. Conch. 40(1): 79-90.

Kozłowski J., Kozłowski R.J. (2011): Exspansion of the invasive slug species Arion lusitanicus Mabille, 1868 (Gastropoda: Pulmonata: Stylommatophora) and dangers to garden crops - a literature review with some new data. Folia Malacologica 19(4): 249-258.

Morrison D.F. (2005): Multivariate statistical methods. Duxbury Press, New York.

Rae R.G., Robertson J.F., Wilson M.J. (2006): The chemotactic response of Phasmarhabditis hermaphrodita (Nematoda: Rhabditidae) to cues of Deroceras reticulatum. Nematology 8: 197-200.

Rae R.G., Robertson J.F., Wilson M.J. (2008): Susceptibility and immune response of Deroceras reticulatum, Milax gagtates and Limax pseudoflavus. J. Invertebrate Pathol. 97: 61-69.

Rae R.G., Robertson J.F., Wilson M.J. (2009): Optimization of biological (Phasmarhabditis hermaphrodita) and chemical (iron phosphate and metaldehyde) slug control in Crop Prot. 28: 765-773.

Scheffe H. (1959): The Analysis of Variance. J. Wiley, New York.

Speiser B., Zaller J.G., Newdecker A. (2001): Size-specific susceptibility of the pest slugs Deroceras reticulatum and Arion lusitanicus to the nematode biocontrol agent Phasmarhabditis hermaphrodita. Biocontrol 46: 311-320.

Tan L., Grewal P.S. (2001): Infection behaviour of the rhabditid nematode Phasmarhabditis hermaphrodita to the grey garden slug Deroceras reticualtum. J. Parasitol. 87: 1349-1354.

Vernavá M.N., Phillps-Aalten P.M., Hughes L.A., Rowcliffe H., Wiltshire C.W., Glen D.M. (2004): Influences of preceding cover crops on slug damage and biological control using Phasmarhabditis hermaphrodita. Ann. Appl. Biol. 145: 279-284. 